\title{
Predictive Capabilities of Three Widely Used Pathology Classification Systems and a Simplified Classification (Beijing Classification) in Primary IgA Nephropathy
}

\author{
Shu-Wei Duan Yan Mei Jian Liu Pu Chen Ping Li Yi-Zhi Chen \\ Shu-Peng Lin Xue-Guang Zhang Jiao-Na Liu Xue-Feng Sun \\ Yuan-Sheng Xie Guang-Yan Cai Shu-Wen Liu Jie Wu Xiang-Mei Chen \\ Department of Nephrology, Chinese PLA General Hospital, Chinese PLA Institute of \\ Nephrology, State Key Laboratory of Kidney Diseases, National Clinical Research Center for \\ Kidney Diseases, Beijing, China
}

\author{
Keywords \\ Classification · IgA nephropathy · Prognosis · Renal pathology
}

\begin{abstract}
Background/Aims: Several pathological classification systems were commonly used in clinical practice to predict the prognosis of IgA nephropathy (IgAN). However, how prognostic value differs between these systems is unclear. The aim of this study was to compare the Lee grade, the Oxford classification, and the Haas classification and to find a simplified classification. Methods: We retrospectively analyzed IgAN cases diagnosed between January 2002 and December 2007. The end points were progression to end-stage renal disease (ESRD) or a $\geq 50 \%$ decline in estimated glomerular filtration rate (eGFR). The predictive capabilities were evaluated by comparing the ability of discrimination (continuous net reclassification) and calibration (Akaike information criterion [AIC]). Results: A total of 412 IgAN patients were included in the study. The average follow-up period was $80.62 \pm 23.63$ months. A total of $44(10.68 \%)$ patients progressed to ESRD, and $70(16.99 \%)$ patients showed a $\geq 50 \%$ decline in eGFR. All multivariate Cox regression models had limited power for high AIC values. The prognostic values of the Lee grade and the Oxford classification were higher than those of models containing only established baseline clinical indicators for progression to ESRD or a $\geq 50 \%$ decline
\end{abstract}

Shu-Wei Duan, Yan Mei, and Jian Liu contributed equally to the conduct and report of this study. 


\section{Kidney \\ Blood Pressure \\ Research}

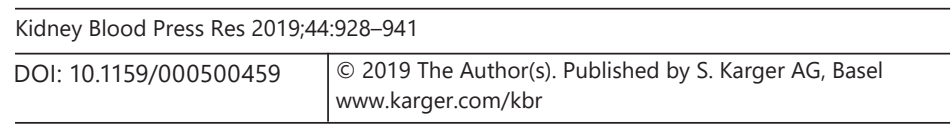

Duan et al.: Comparing Pathological Classifications of IgA Nephropathy

in eGFR (Lee grade $0.50,95 \% \mathrm{Cl} 0.21-0.74$; Oxford classification $0.48,95 \% \mathrm{Cl} 0.28-0.71$ ). The prognostic value of the Haas classification was lower than that of the other pathological classification systems for progression to ESRD or a $\geq 50 \%$ decline in eGFR (Lee grade $0.53,95 \% \mathrm{Cl}$ 0.23-0.92; Oxford classification $0.59,95 \% \mathrm{Cl} 0.10-0.74)$. The prognostic value of hierarchical classification (Beijing classification) using $\mathrm{M}$ and $\mathrm{T}$ lesion was similar to the Oxford classification. Conclusions: Both the Lee grade and the Oxford classification showed incremental prognostic values beyond established baseline clinical indicators. The Haas classification was slightly inferior to the Lee grade and the Oxford classification. The hierarchical classification (Beijing classification) using less pathological parameters does not lose predictive efficiency.

(c) 2019 The Author(s)

Published by S. Karger AG, Basel

\section{Introduction}

IgA nephropathy (IgAN) is the most common primary kidney disease and leading cause of end-stage renal disease (ESRD) in China $[1,2]$. The characteristic and diagnostic lesion of IgAN is glomerular IgA deposition, as a unique or predominant positive immunoglobulin. However, the clinical course and pathological lesions of IgAN are highly variable [3, 4]. Pathological lesions of IgAN are so complicated that a number of researchers have developed pathological classification systems, of which there are two basic types: single grade, such as the Lee grade [5] and the Haas classification [6], and semiquantitative grade, such as the Oxford classification system [7], which was updated recently [8]. The Lee grade, one of the early editions of widespread pathological classification systems, was proposed in 1982. Considering the candidate prognostic histological lesions comprehensively, the Lee grade was characteristically simple to operate and easy to apply. Based on the Lee grade for IgAN and the World Health Organization classification system for lupus nephritis [9], the Haas classification was published in 1997. Focal-segmental glomerulosclerosis-like lesions were listed as a sole subclass, and lesions of tubular atrophy or loss of $>40 \%$ of renal cortex were identified as the worst subclass, regardless of glomerular lesions. The Oxford classification was presented by an international consensus working group, an informal network of nephrologists and pathologists, in 2007. It defined the pathological lesions for the first time and compared the correlations and reproducibility of different lesions [10]. Although there are many studies evaluating the prognostic value of the three different pathological classification systems [11-13], comparative analysis has been scarce [14]. Therefore, this study aimed to compare the prognostic values of the three pathological classification systems in primary IgAN in a retrospective cohort.

\section{Subjects and Methods}

\section{Patients}

We retrospectively examined patient records for new cases of IgAN that were confirmed via biopsy between January 2002 and December 2007 in the Department of Nephrology, Chinese PLA General Hospital. We collated details of hospital admissions, open clinic visits, and primary and secondary diagnoses based on the International Classification of Diseases, 9th edition. Inclusion criteria for the study were being newly diagnosed with IgAN, being aged $\geq 14$ years, and having an estimated glomerular filtration rate (eGFR) of $\geq 15 \mathrm{~mL} / \mathrm{min} /$ $1.73 \mathrm{~m}^{2}$ and no less than eight glomeruli available at biopsy. Exclusion criteria for the study were presence of secondary IgAN, comorbidity with systemic diseases such as diabetes mellitus, or a follow-up time of $<36$ months. 


\section{Kidney \\ Blood Pressure \\ Research}

\begin{tabular}{l|l}
\hline Kidney Blood Press Res 2019:44:928-941 \\
\hline DOI: 10.1159/000500459 & $\begin{array}{l}\text { @ 2019 The Author(s). Published by S. Karger AG, Basel } \\
\text { www.karger.com/kbr }\end{array}$ \\
\hline
\end{tabular}

Duan et al.: Comparing Pathological Classifications of IgA Nephropathy

\section{Measurement}

We evaluated sex, age at biopsy, baseline proteinuria, baseline systolic and diastolic blood pressure, and serum creatinine within a week of biopsy. Mean arterial pressure (MAP) was estimated as diastolic pressure plus one-third pulse pressure. Serum creatinine was measured using a Roche MODULAR automatic biochemistry analyzer. Patients' eGFR was calculated using the Chronic Kidney Disease Epidemiology Collaboration equation adjusted for Asian populations [15]. Pathological lesions were rescored independently, using light microscopy, by two experienced pathologists according to the Lee grade, the Haas classification, and the Oxford classification. Five lesions were included in the Oxford classification: mesangial hypercellularity (M); endocapillary hypercellularity (E); segmental glomerulosclerosis (S), including any amount of the tuft involved in sclerosis, but not involving the whole tuft, or the presence of an adhesion; tubular atrophy/interstitial fibrosis (T); and crescents (C) according to the updated Oxford classification [8]. M is scored for each glomerulus by assessing the most cellular mesangial area, excluding mesangial areas adjacent to the vascular stalk. Less than 4 mesangial cells/mesangial area is scored as 0, 4-5 mesangial cells/mesangial area is scored as 1, 6-7 mesangial cells/ mesangial area is scored as 2 , and $\geq 8$ mesangial cells/mesangial area is scored as 3 . The mean mesangial score is the total of the mesangial scores divided by the number of scorable glomeruli. $\mathrm{M} 0 / \mathrm{M} 1$ is defined as mesangial hypercellularity score $\leq 0.5 />0.5$. E0/E1 is defined as any endocapillary hypercellularity absent/present. S0/S1 is defined as the absence/presence of segmental glomerulosclerosis or adhesion. T0/T1/T2 is defined as $<25 \% / 26-50 \% />50 \%$ tubular atrophy/ interstitial fibrosis. C0/C1/C2 is defined as absence $/<25 \% / \geq 25 \%$ of crescents, including cellular and fibrocellular crescents. Follow-up patient data were collected via telephone interview. The use of renin-angiotensin system blockade, corticosteroids, and immunosuppressants during follow-up was reported. Renin-angiotensin system blockade indicated exposure to angiotensinconverting enzyme inhibitors, angiotensin receptor blockers, or both. The following immunosuppressive drugs were recorded: cyclophosphamide, cyclosporine, tacrolimus, mycophenolate mofetil, leflunomide, and Tripterygium wilfordii. The start point was the time of biopsy. The endpoint was the requirement for renal replacement therapy, identified as progression to ESRD (eGFR $<15 \mathrm{~mL} / \mathrm{min} / 1.73 \mathrm{~m}^{2}$ ) or a $\geq 50 \%$ decline in eGFR. Serum creatinine and endpoint events were measured. All data entry and telephone interviews were performed by an independent clinical reviewer prior to February 1, 2014.

\section{Statistical Methods}

The Kolmogorov-Smirnov test was used to determine the normality of the distribution of continuous variables. Continuous variables with normal and nonnormal distribution were expressed as means (standard deviations) and medians (interquartile ranges), respectively. Cutoffs for continuous variables were determined according to professional knowledge and published articles. Categorical variables were expressed as absolute values and percentages. The kappa coefficient was used to evaluate differences in scoring pathology between different pathologists; kappa $\geq 0.6$ shows good concordance. If the results of the two pathologists were different, it was determined by discussion. Univariate analysis was performed using the univariate Cox survival analysis to identify candidate predictors, taking into consideration established prognostic clinical indicators such as sex, age, MAP, initial proteinuria, eGFR, and chronic kidney disease stage as well as the three pathological classification systems. Multivariate Cox survival regression models were used to identify independent clinical predictors of the endpoints. Variables associated with the endpoints received further consideration for multivariate Cox survival analysis. In the third step, by adjusting for independent clinical predictors, three different multivariate Cox survival regression models were constructed for the three pathological classification systems. The goodness of fit of the Cox survival regression models was evaluated via Akaike information criterion (AIC). Models with smaller AIC or $\chi^{2}$ values are called well calibrated. 
Kidney

Blood Pressure

Research

Table 1. Clinicopathological features at the time of biopsy $(n=412)$

\begin{tabular}{l|l}
\hline Kidney Blood Press Res 2019;44:928-941 \\
\hline DOI: 10.1159/000500459 & $\begin{array}{l}\text { ○ 2019 The Author(s). Published by S. Karger AG, Basel } \\
\text { www.karger.com/kbr }\end{array}$ \\
\hline
\end{tabular}

Duan et al.: Comparing Pathological Classifications of IgA Nephropathy

\begin{tabular}{|c|c|}
\hline \multicolumn{2}{|l|}{ Features } \\
\hline Age, years & $34.95 \pm 10.79$ \\
\hline$\leq 20$ & $43(10.44 \%)$ \\
\hline $21-30$ & $104(25.24 \%)$ \\
\hline $31-40$ & $137(33.25 \%)$ \\
\hline $41-50$ & $98(23.79 \%)$ \\
\hline $51-60$ & $24(5.83 \%)$ \\
\hline$>60$ & $6(1.46 \%)$ \\
\hline Male sex & $223(54.13 \%)$ \\
\hline Proteinuria, g/24 h & $0.89(0.48-1.79)$ \\
\hline MAP, mm Hg & $91.86 \pm 11.59$ \\
\hline $\mathrm{eGFR}, \mathrm{mL} / \mathrm{min} / 1.73 \mathrm{~m}^{2}$ & 97.10 (71.78-119.87) \\
\hline \multicolumn{2}{|l|}{ CKD stage } \\
\hline 1 & $241(58.50 \%)$ \\
\hline 2 & $109(26.46 \%)$ \\
\hline $3 a$ & $28(6.80 \%)$ \\
\hline $3 b$ & $28(6.80 \%)$ \\
\hline 4 & $6(1.46 \%)$ \\
\hline \multicolumn{2}{|l|}{ Lee grade } \\
\hline I & $7(1.70 \%)$ \\
\hline II & $41(9.95 \%)$ \\
\hline III & $238(57.77 \%)$ \\
\hline IV & $102(24.76 \%)$ \\
\hline V & $24(5.83 \%)$ \\
\hline \multicolumn{2}{|l|}{ Haas classification } \\
\hline $\mathrm{I}$ & $40(9.71 \%)$ \\
\hline II & $20(4.85 \%)$ \\
\hline III & $281(68.20 \%)$ \\
\hline IV & $27(6.55 \%)$ \\
\hline V & $44(10.68 \%)$ \\
\hline \multicolumn{2}{|l|}{ Oxford classification } \\
\hline M1 & $174(42.23 \%)$ \\
\hline E1 & $58(14.08 \%)$ \\
\hline S1 & $294(71.36 \%)$ \\
\hline \multicolumn{2}{|r|}{ - } \\
\hline T0 & $55(13.35 \%)$ \\
\hline $\mathrm{T} 1$ & $255(61.89 \%)$ \\
\hline $\mathrm{T} 2$ & $102(24.76 \%)$ \\
\hline \multicolumn{2}{|r|}{$e^{-1}(-100)$} \\
\hline $\mathrm{CO}$ & 339 (82.28\%) \\
\hline $\mathrm{C} 1$ & $71(17.23 \%)$ \\
\hline $\mathrm{C} 2$ & $2(0.49 \%)$ \\
\hline
\end{tabular}

C, crescents; CKD, chronic kidney disease; E, endocapillary hypercellularity; eGFR, estimated glomerular filtration rate; $\mathrm{M}$, mesangial hypercellularity; MAP, mean arterial pressure; S, segmental glomerulosclerosis; T, tubular atrophy/interstitial fibrosis.

Determination of whether one set of discrimination abilities was superior to another was made using the continuous net reclassification improvement (NRI) described in Pencina et al. $\mathrm{NRI}(>0)=2 \times[\mathrm{P}($ up $\mid$ event $)-\mathrm{P}($ up $\mid$ nonevent $)][16]$. The predicted time horizon was estimated by extrapolating the observed rates in the Kaplan-Meier survival analysis. Censoring was adjusted by the inverse probability censoring weight. Its magnitude is more important than statistical significance. Presenting NRI with its $\mathrm{CI}$ is recommended. All $p$ values were two-tailed, and values $<0.05$ were considered statistically significant. Statistical analyses were performed using SPSS software (version 17.0; SPSS Inc., Chicago, IL, USA) and R programming (version 3.1.0). 


\section{Kidney \\ Blood Pressure \\ Research}

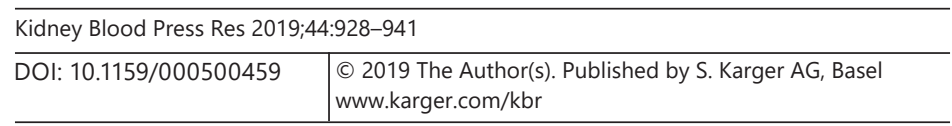

Duan et al.: Comparing Pathological Classifications of IgA Nephropathy
Table 2. Kappa coefficient of the Lee grade, the Haas classification, and the Oxford classification

\begin{tabular}{lll}
\hline Classification & Kappa coefficient & $95 \%$ CI \\
\hline Lee grade & 0.76 & $0.70-0.82$ \\
Haas classification & 0.83 & $0.76-0.90$ \\
Oxford classification & & \\
M & 0.77 & $0.70-0.84$ \\
E & 0.74 & $0.64-0.82$ \\
S & 0.86 & $0.79-0.93$ \\
T & 0.86 & $0.82-0.90$ \\
C & 0.84 & $0.77-0.88$ \\
\hline
\end{tabular}

C, crescents; E, endocapillary hypercellularity; M, mesangial hypercellularity; S, segmental glomerulosclerosis; T, tubular atrophy/interstitial fibrosis.

\section{Results}

Of 483 IgAN patients who met the inclusion and exclusion criteria, 71 were not followed and were excluded; 412 cases were included in the study. Patient characteristics at biopsy are presented in Table 1. The patients' mean age was $34.95 \pm 10.79$ years, and $223(54.13 \%)$ patients were men. Renal impairment (eGFR $<60 \mathrm{~mL} / \mathrm{min} / 1.73 \mathrm{~m}^{2}$ ) was present in $62(15.06 \%)$ patients. The most common histological classification was present in $238(57.77 \%)$ patients with Lee grade III and 281 (68.20\%) patients with Haas classification III. The most common histological lesions in the Oxford classification were S1 and T1 lesions, present in 294 (71.36\%) and $255(61.89 \%)$ patients, respectively. The kappa coefficients of the three pathological classifications were all $>0.6$, suggesting that their repeatability was good (Table 2).

The average follow-up period was $80.62 \pm 23.63$ months, and 4 patients died. A total of $44(10.68 \%)$ patients progressed to ESRD, and 70 (16.99\%) showed a $\geq 50 \%$ decline in eGFR (including progression to ESRD).

The results of univariate Cox survival analysis showed an association between clinicopathological features and progression to ESRD or a $\geq 50 \%$ decline in eGFR (Table 3 ). Univariate analysis revealed that proteinuria, MAP, eGFR, chronic kidney disease stage, Lee grade, Haas classification, and $\mathrm{M}, \mathrm{S}$, and T lesions of the Oxford classification significantly correlated with progression to ESRD or a $\geq 50 \%$ decline in eGFR $(p<0.05)$. In addition, age, sex, and E and C lesions of the Oxford classification were not predictive of progression to ESRD or a $\geq 50 \%$ decline in eGFR $(p>0.05)$. The results of multivariate Cox survival analysis indicated an association between clinical features and progression to ESRD or a $\geq 50 \%$ decline in eGFR (Table 4). In the multivariate analysis, the independent clinical predictors for ESRD or a $\geq 50 \%$ decline in eGFR were proteinuria $(1.19,95 \%$ CI 1.08-1.30), MAP $(1.57,95 \%$ CI 1.33-1.86), and eGFR $(0.78,95 \%$ CI 0.71-0.85). Three multivariate Cox survival models were used to analyze the association between pathological features and progression to ESRD or a $\geq 50 \%$ decline in eGFR after adjusting for clinical features (Table 5). The pathological lesions determined by Lee grade were correlated with progression to ESRD or a $\geq 50 \%$ decline in eGFR $(p<0.001)$ after adjusting for baseline clinical indicators. Relative to Lee grade I and II, the risk of progression to ESRD or a $\geq 50 \%$ decline was 4.21 (95\% CI 0.93-18.98) for Lee grade III, 12.94 (95\% CI 2.84-58.99) for grade IV, and 36.76 (95\% CI 7.14-189.25) for Lee grade V. Relative to Haas classifications I, II, and III, the risk of progression to ESRD or a $\geq 50 \%$ decline in eGFR was 3.68 (95\% CI 1.87-7.22) in Haas classification IV and 3.67 (95\% CI 1.79-7.52) in Haas classification V. In the Oxford classification, M $(2.81,95 \%$ CI 1.52-5.21) and T $(3.11,95 \%$ 


\section{Research}

Duan et al.: Comparing Pathological Classifications of IgA Nephropathy

Table 3. Univariate Cox survival analysis of the association between clinicopathological features and progression to ESRD or $\geq 50 \%$ decline in eGFR

\begin{tabular}{|c|c|c|c|c|}
\hline \multirow[t]{2}{*}{ Variables } & \multicolumn{2}{|l|}{ ESRD } & \multicolumn{2}{|c|}{ ESRD or $\geq 50 \%$ decline in eGFR } \\
\hline & HR $(95 \%$ CI $)$ & $p$ & HR (95\% CI) & $p$ \\
\hline Male sex & $1.79(0.95-3.39)$ & 0.071 & $1.10(0.68-1.76)$ & 0.704 \\
\hline Age, every 1 year & $1.00(0.98-1.03)$ & 0.859 & $1.01(0.99-1.04)$ & 0.180 \\
\hline Proteinuria & $1.22(1.13-1.32)$ & $<0.001$ & $1.16(1.08-1.25)$ & $<0.001$ \\
\hline MAP, every 10 mm Hg & $1.70(1.38-2.09)$ & $<0.001$ & $1.61(1.36-1.91)$ & $<0.001$ \\
\hline eGFR, every $10 \mathrm{~mL} / \mathrm{min} / 1.73 \mathrm{~m}^{2}$ & $0.74(0.67-0.83)$ & $<0.001$ & $0.77(0.71-0.84)$ & $<0.001$ \\
\hline CKD stage & & $<0.001$ & & $<0.001$ \\
\hline 1 & - & - & - & - \\
\hline 2 & $3.41(1.53-7.60)$ & 0.003 & $2.36(1.33-4.19)$ & 0.003 \\
\hline $3 a$ & $10.61(4.14-27.19)$ & $<0.001$ & $6.56(2.99-14.37)$ & $<0.001$ \\
\hline $3 b$ & $9.95(3.90-25.40)$ & $<0.001$ & $6.43(3.11-13.30)$ & $<0.001$ \\
\hline 4 & $28.17(7.62-104.22)$ & $<0.001$ & $16.24(4.78-55.17)$ & $<0.001$ \\
\hline Lee grade & & $<0.001$ & & $<0.001$ \\
\hline I-II & - & - & - & - \\
\hline III & $3.55(0.46-27.25)$ & 0.222 & $3.22(0.75-13.80)$ & 0.116 \\
\hline IV & 13.11 (1.74-98.87) & 0.013 & $16.50(3.93-69.24)$ & $<0.001$ \\
\hline $\mathrm{V}$ & $52.68(6.79-408.78)$ & $<0.001$ & 43.09 (9.68-191.74) & $<0.001$ \\
\hline Haas classification & & $<0.001$ & & $<0.001$ \\
\hline I-III & - & - & - & - \\
\hline IV & $5.17(2.14-12.46)$ & $<0.001$ & $4.75(2.50-9.01)$ & $<0.001$ \\
\hline V & $14.37(7.47-27.64)$ & $<0.001$ & $8.95(5.24-15.29)$ & $<0.001$ \\
\hline \multicolumn{5}{|l|}{ Oxford classification } \\
\hline M1 & 7.80 (3.61-16.83) & $<0.001$ & $6.18(3.56-10.72)$ & $<0.001$ \\
\hline E1 & $0.63(0.22-1.76)$ & 0.376 & $0.79(0.36-1.74)$ & 0.562 \\
\hline S1 & $6.09(1.88-19.66)$ & 0.003 & $2.78(1.42-5.44)$ & 0.003 \\
\hline $\mathrm{C}$ & & 0.257 & & 0.442 \\
\hline $\mathrm{CO}$ & - & - & - & - \\
\hline $\mathrm{C} 1$ & $1.24(0.59-2.58)$ & 0.207 & $1.25(0.69-2.25)$ & 0.450 \\
\hline $\mathrm{C} 2$ & $5.75(0.78-42.37)$ & 0.086 & $4.93(0.67-36.07)$ & 0.116 \\
\hline $\mathrm{T} 2$ (vs. T0+T1) & $6.55(3.53-12.18)$ & $<0.001$ & $7.59(4.64-12.42)$ & $<0.001$ \\
\hline
\end{tabular}

C, crescents; CKD, chronic kidney disease; E, endocapillary hypercellularity; eGFR, estimated glomerular filtration rate; ESRD, end-stage renal disease; M, mesangial hypercellularity; MAP, mean arterial pressure; S, segmental glomerulosclerosis; T, tubular atrophy/interstitial fibrosis.

Table 4. Multivariate Cox survival analysis of the association between clinical features and progression to ESRD or $\geq 50 \%$ decline in eGFR

\begin{tabular}{lll}
\hline Variables & \multicolumn{2}{l}{ ESRD or $\geq 50 \%$ decline in eGFR } \\
\cline { 3 - 3 } & HR $(95 \% \mathrm{CI})$ & $p$ \\
\hline Proteinuria & $1.19(1.08-1.30)$ & $<0.001$ \\
MAP (every $10 \mathrm{~mm} \mathrm{Hg})$ & $1.57(1.33-1.86)$ & $<0.001$ \\
eGFR (every $\left.10 \mathrm{~mL} / \mathrm{min} / 1.73 \mathrm{~m}^{2}\right)$ & $0.78(0.71-0.85)$ & $<0.001$ \\
\hline
\end{tabular}

eGFR, estimated glomerular filtration rate; ESRD, end-stage renal disease; MAP, mean arterial pressure. 
Table 5. Multivariate Cox survival regression models analysis of the association between pathological features and progression to ESRD or $\geq 50 \%$ decline in eGFR according to the Lee grade, the Haas classification, and the Oxford classification after adjusting clinical features

\begin{tabular}{|c|c|c|}
\hline \multirow[t]{2}{*}{ Variables } & \multicolumn{2}{|c|}{ ESRD or $\geq 50 \%$ decline in eGFR } \\
\hline & HR $(95 \%$ CI $)$ & $p$ \\
\hline \multicolumn{3}{|l|}{ Lee grade model } \\
\hline Proteinuria & $1.21(1.09-1.35)$ & 0.001 \\
\hline MAP, every 10 mm Hg & $1.45(1.21-1.73)$ & $<0.001$ \\
\hline eGFR, every $10 \mathrm{~mL} / \mathrm{min} / 1.73 \mathrm{~m}^{2}$ & $0.94(0.84-1.04)$ & 0.225 \\
\hline Lee grade & & $<0.001$ \\
\hline I+II & - & - \\
\hline III & $4.21(0.93-18.98)$ & 0.062 \\
\hline IV & $12.94(2.84-58.99)$ & 0.001 \\
\hline $\mathrm{V}$ & $36.76(7.14-189.25)$ & $<0.001$ \\
\hline \multicolumn{3}{|l|}{ Haas classification model } \\
\hline Proteinuria & $1.15(1.04-1.27)$ & 0.006 \\
\hline MAP, every 10 mm Hg & $1.49(1.26-1.78)$ & $<0.001$ \\
\hline eGFR, every $10 \mathrm{~mL} / \mathrm{min} / 1.73 \mathrm{~m}^{2}$ & $0.88(0.79-0.98)$ & 0.016 \\
\hline Haas classification & & $<0.001$ \\
\hline I+II+III & - & - \\
\hline IV & $3.68(1.87-7.22)$ & $<0.001$ \\
\hline $\mathrm{V}$ & $3.67(1.79-7.52)$ & $<0.001$ \\
\hline \multicolumn{3}{|l|}{ Oxford classification model } \\
\hline Proteinuria & $1.12(1.01-1.25)$ & 0.035 \\
\hline MAP, every $10 \mathrm{~mm} \mathrm{Hg}$ & $1.40(1.17-1.68)$ & $<0.001$ \\
\hline eGFR, every $10 \mathrm{~mL} / \mathrm{min} / 1.73 \mathrm{~m}^{2}$ & $0.91(0.82-1.00)$ & 0.055 \\
\hline \multicolumn{3}{|l|}{ Oxford classification } \\
\hline M & $2.81(1.52-5.21)$ & 0.001 \\
\hline $\mathrm{T} 2$ (vs. T0+T1) & $3.11(1.67-5.81)$ & $<0.001$ \\
\hline
\end{tabular}

eGFR, estimated glomerular filtration rate; ESRD, end-stage renal disease; M, mesangial hypercellularity; MAP, mean arterial pressure; T, tubular atrophy/interstitial fibrosis.

Table 6. Corticosteroids or other immunosuppressive agents in patients with $\mathrm{E} / \mathrm{C}$ lesions

\begin{tabular}{lrrrrrrr}
\hline \multirow{2}{*}{ Variables } & \multicolumn{2}{c}{ Corticosteroids } & \multirow{2}{*}{$p$} & \multicolumn{2}{c}{ Other IS } & \multirow{2}{*}{$p$} \\
\cline { 2 - 3 } \cline { 5 - 6 } & no & yes & & no & yes & \\
\hline E0 & 244 & 110 & & 0.016 & 193 & 161 & 0.776 \\
E1 & 30 & 28 & & 30 & 28 & \\
C0 & 246 & 93 & & $<0.001$ & 194 & 145 & 0.004 \\
C1+C2 & 28 & 45 & & 29 & 44 & \\
\hline
\end{tabular}

C, crescents; E, endocapillary hypercellularity; IS, immunosuppressive agents.

CI 1.67-5.81) lesions were independent risk factors for progression to ESRD or a $\geq 50 \%$ decline in eGFR. The $\mathrm{S}, \mathrm{E}$, and $\mathrm{C}$ lesions of the Oxford classification did not correlate with prognosis $(p>0.05)$. In addition, patients with $\mathrm{E}$ or $\mathrm{C}$ lesions have a higher probability to receive corticosteroids $(p<0.01)$. Patients with $\mathrm{C}$ lesion have a higher probability to receive other immunosuppressive agents $(p<0.01)$ (Table 6). 
Table 7. Pathological features between the three classification systems

\begin{tabular}{|c|c|c|c|c|c|c|c|c|c|c|c|c|c|}
\hline & & \multicolumn{12}{|c|}{ Oxford classification } \\
\hline & & M & M & E0 & E1 & So & S1 & $\mathrm{T}$ & $\mathrm{T}$ & $\mathrm{T}$ & $\mathrm{CO}$ & $\mathrm{C} 1$ & $\mathrm{C} 2$ \\
\hline \multirow[t]{5}{*}{ Lee grade } & I & 0 & 1 & 7 & 0 & 7 & 0 & 0 & 1 & 2 & 7 & 0 & 0 \\
\hline & II & 37 & 4 & 38 & 3 & 31 & 10 & 24 & 17 & 0 & 38 & 3 & 0 \\
\hline & III & 157 & 81 & 196 & 42 & 61 & 177 & 24 & 194 & 20 & 201 & 36 & 1 \\
\hline & IV & 30 & 72 & 91 & 11 & 14 & 88 & 0 & 44 & 58 & 73 & 29 & 0 \\
\hline & $\mathrm{V}$ & 7 & 17 & 22 & 2 & 5 & 19 & 0 & 0 & 24 & 20 & 3 & 1 \\
\hline Haas & I & 39 & 1 & 35 & 5 & 35 & 5 & 23 & 17 & 0 & 40 & 0 & 0 \\
\hline \multirow[t]{4}{*}{ classification } & II & 17 & 3 & 20 & 0 & 5 & 15 & 5 & 15 & 0 & 19 & 1 & 0 \\
\hline & III & 167 & 114 & 234 & 47 & 67 & 214 & 26 & 212 & 43 & 229 & 51 & 1 \\
\hline & IV & 4 & 23 & 23 & 4 & 4 & 23 & 1 & 6 & 20 & 18 & 9 & 0 \\
\hline & V & 11 & 33 & 42 & 2 & 7 & 37 & 0 & 5 & 39 & 33 & 10 & 1 \\
\hline Total & & 238 & 174 & 354 & 58 & 118 & 294 & 55 & 255 & 102 & 339 & 71 & 2 \\
\hline
\end{tabular}

C, crescents; E, endocapillary hypercellularity; M, mesangial hypercellularity; S, segmental glomerulosclerosis; T, tubular atrophy/interstitial fibrosis.

Table 8. Correlation analysis between the three classifications

\begin{tabular}{llrrrrr}
\hline Classification & Statistics & Oxford M & Oxford E & Oxford S & Oxford T & Oxford C \\
\hline Lee grade & CC & 0.411 & -0.023 & 0.331 & 0.688 & 0.157 \\
& $p$ & $<0.001$ & 0.645 & $<0.001$ & $<0.001$ & 0.001 \\
\hline Haas classification & CC & 0.411 & -0.016 & 0.316 & 0.615 & 0.190 \\
& $p$ & $<0.001$ & 0.744 & $<0.001$ & $<0.001$ & $<0.001$ \\
\hline
\end{tabular}

C, crescents; CC, correlation coefficient; E, endocapillary hypercellularity; M, mesangial hypercellularity; $\mathrm{S}$, segmental glomerulosclerosis; $\mathrm{T}$, tubular atrophy/interstitial fibrosis.

Table 9. Log-rank test of different survival curves

\begin{tabular}{|c|c|c|c|c|c|c|c|c|c|c|}
\hline \multirow[t]{2}{*}{ Grade } & \multicolumn{2}{|l|}{ м0т0 } & \multicolumn{2}{|l|}{ M1T0 } & \multicolumn{2}{|l|}{ M0T1 } & \multicolumn{2}{|l|}{ M1T1 } & \multicolumn{2}{|l|}{ М0Т2 } \\
\hline & $\chi^{2}$ & $p$ & $\chi^{2}$ & $p$ & $\chi^{2}$ & $p$ & $\chi^{2}$ & $p$ & $\chi^{2}$ & $p$ \\
\hline M0T0 & - & - & & & & & & & & \\
\hline M1T0 & 0.73 & 0.068 & - & - & & & & & & \\
\hline M0T1 & 39.14 & $<0.001$ & 5.34 & 0.015 & - & - & & & & \\
\hline M1T1 & 149.69 & $<0.001$ & 26.28 & $<0.001$ & 19.07 & $<0.001$ & - & - & & \\
\hline M0T2 & 31.94 & $<0.001$ & 4.22 & 0.040 & 26.23 & $<0.001$ & 0.987 & 0.320 & - & - \\
\hline M1T2 & 137.35 & $<0.001$ & 34.27 & $<0.001$ & 88.68 & $<0.001$ & 19.610 & $<0.001$ & 3.563 & 0.029 \\
\hline
\end{tabular}

M, mesangial hypercellularity; T, tubular atrophy/interstitial fibrosis.

Correlation of different classifications was shown (Tables 7, 8). The proportion of Oxford M1 S1 T1 C1 was increased along with the rise of Lee grade and Haas classification. However, Oxford E1 did not show the same tendency. Correlation analysis showed that Oxford M/S/T/C was positively correlated with Lee grade and Haas classification. 
Fig. 1. Kaplan-Meier survival curves based on $\mathrm{M}$ and $\mathrm{T}$ lesions.

Fig. 2. Kaplan-Meier survival curves of the simplified pathological system.
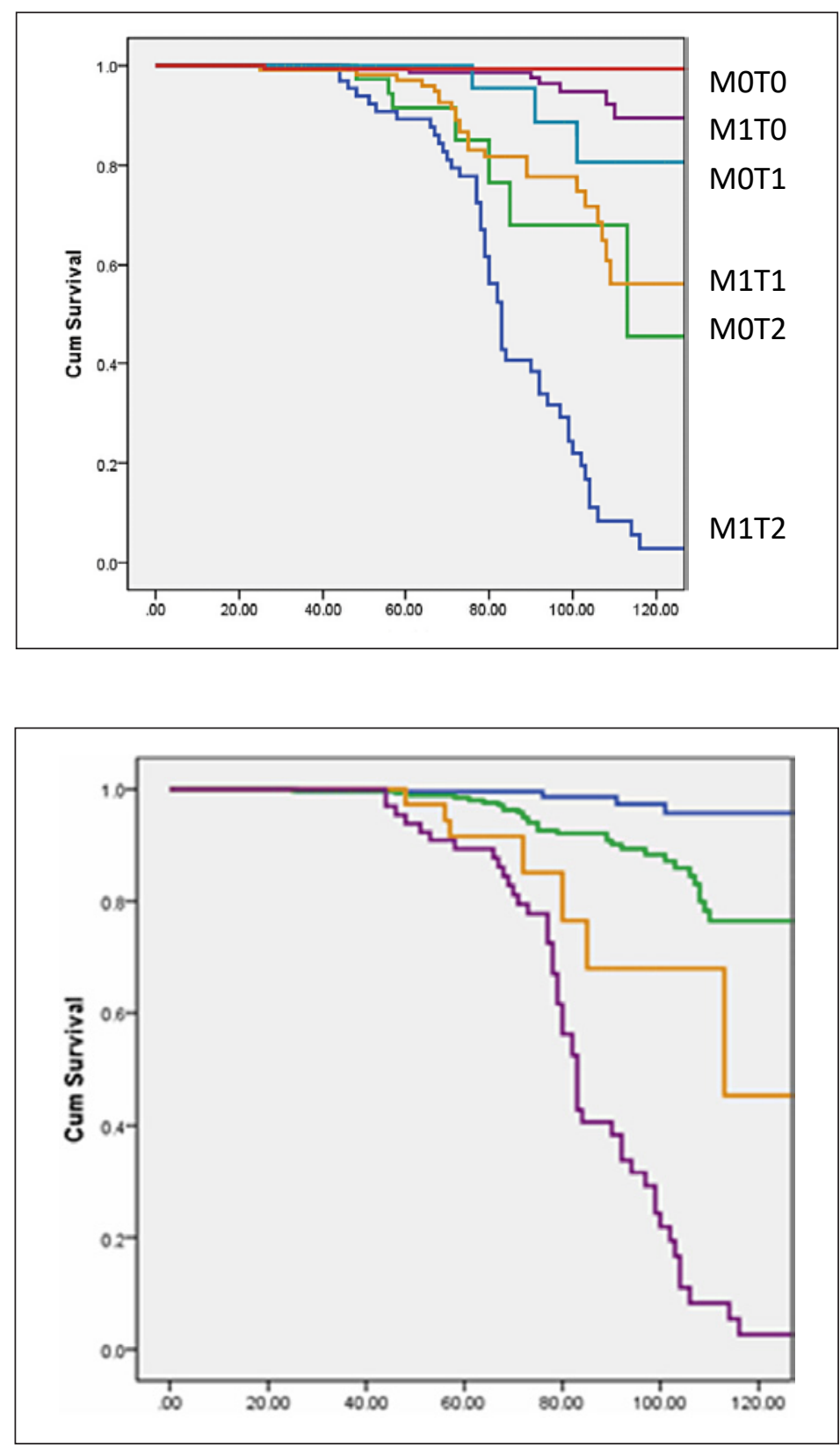

Multivariate Cox survival analysis (Table 4) showed that clinical indicators such as baseline proteinuria, MAP, and eGFR were prognostic factors of endpoints. After adjusting for those clinical indicators, Lee grade, Haas classification, and M/T lesion of the Oxford classification were independent risk factors of endpoints (Table 5).

A simplified classification system was made by $\mathrm{M}$ and $\mathrm{T}$ lesion. There were some intersections of survival curves with varying degrees of pathological changes (Fig. 1). By log-rank test (Table 9), intertwined curves ( $p>0.05$ ) were merged into one. The final simplified classification (Beijing classification) was shown (Table 10). Grade 1 is M0T0 or M1T0, grade 2 is M0T1, grade 3 is M1T1 or M0T2, and grade 4 is M1T2. There are no crossed survival curves between different grades (Fig. 2).

The Cox survival regression analysis results, including AIC and $\chi^{2}$, are presented (Table 11). The AIC values for models of progression to ESRD or a $\geq 50 \%$ decline in eGFR were 
Table 10. The new simplified pathological system (Beijing classification)

\begin{tabular}{ll}
\hline Grade & Pathological parameters \\
\hline 1 & M0T0 or M1T0 \\
2 & M0T1 \\
3 & M1T1 or M0T2 \\
4 & M1T2 \\
\hline
\end{tabular}

M, mesangial hypercellularity; $\mathrm{T}$, tubular atrophy/interstitial fibrosis.

Table 11. Fit of multivariate Cox survival regression models analysis of the association between pathological features and progression to ESRD or $\geq 50 \%$ decline in eGFR according to the Lee grade, the Haas classification, and the Oxford classification after adjusting clinical features

\begin{tabular}{|c|c|c|c|c|c|}
\hline \multirow[t]{2}{*}{ Models } & \multicolumn{4}{|c|}{ ESRD or $\geq 50 \%$ decline in eGFR } & \multirow{2}{*}{$\begin{array}{l}\text { Simplified } \\
\text { classification }\end{array}$} \\
\hline & $\begin{array}{l}\text { clinical } \\
\text { model }\end{array}$ & $\begin{array}{l}\text { Lee } \\
\text { grade }\end{array}$ & $\begin{array}{l}\text { Haas } \\
\text { classification }\end{array}$ & $\begin{array}{l}\text { Oxford } \\
\text { classification }\end{array}$ & \\
\hline AIC & 657.68 & 624.25 & 638.75 & 622.14 & 582 \\
\hline$\chi^{2}$ & 78.90 & 143.22 & 134.56 & 139.75 & 114.32 \\
\hline
\end{tabular}

AIC, Akaike information criterion; eGFR, estimated glomerular filtration rate; ESRD, end-stage renal disease.

Table 12. Reclassification of the predicted 10 -year risk of progression to ESRD or $\geq 50 \%$ decline in eGFR based on clinical and clinicopathological Cox survival regression models according to the Lee grade, the Haas classification, and the Oxford classification

\begin{tabular}{|c|c|c|c|c|c|}
\hline \multirow[t]{2}{*}{ Models } & \multirow[t]{2}{*}{ NRI } & \multicolumn{3}{|c|}{ ESRD or $\geq 50 \%$ decline in eGFR } & \multirow{2}{*}{$\begin{array}{l}\text { Simplified } \\
\text { classification }\end{array}$} \\
\hline & & $\begin{array}{l}\text { Lee } \\
\text { grade }\end{array}$ & $\begin{array}{l}\text { Haas } \\
\text { classification }\end{array}$ & $\begin{array}{l}\text { Oxford } \\
\text { classification }\end{array}$ & \\
\hline \multirow[t]{3}{*}{ Clinical model } & event & 0.75 & 0.65 & 0.77 & 0.78 \\
\hline & nonevent & 0.25 & 0.43 & 0.29 & 0.27 \\
\hline & $95 \% \mathrm{CI}$ & 0.21 to 0.74 & -0.27 to 0.81 & 0.28 to 0.71 & 0.25 to 0.74 \\
\hline \multirow[t]{3}{*}{ Lee grade } & event & - & 0.36 & 0.51 & 0.50 \\
\hline & nonevent & - & 0.89 & 0.46 & 0.44 \\
\hline & $95 \% \mathrm{CI}$ & - & -0.77 to -0.08 & -0.33 to 0.51 & -0.31 to 0.52 \\
\hline \multirow{3}{*}{$\begin{array}{l}\text { Haas } \\
\text { classification }\end{array}$} & event & - & - & 0.73 & 0.78 \\
\hline & nonevent & - & - & 0.14 & 0.15 \\
\hline & $95 \% \mathrm{CI}$ & - & - & 0.10 to 0.74 & 0.12 to 0.78 \\
\hline \multirow{3}{*}{$\begin{array}{l}\text { Oxford } \\
\text { classification }\end{array}$} & event & - & - & - & 0.52 \\
\hline & nonevent & - & - & - & 0.49 \\
\hline & $95 \% \mathrm{CI}$ & - & - & - & -0.36 to 0.51 \\
\hline
\end{tabular}

eGFR, estimated glomerular filtration rate; ESRD, end-stage renal disease; NRI, net reclassification improvement. 


\section{Kidney \\ Blood Pressure \\ Research}

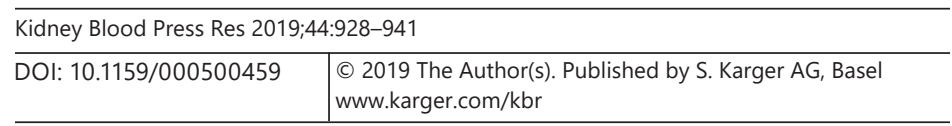

Duan et al.: Comparing Pathological Classifications of IgA Nephropathy

657.68 in the clinical model, 624.25 in the Lee grade model, 638.75 in the Haas classification model, and 622.14 in the Oxford classification model. The $\chi^{2}$ values for models of progression to ESRD or a $\geq 50 \%$ decline in eGFR were 78.90 in the clinical model, 143.22 in the Lee grade model, 134.56 in the Haas classification model, and 139.75 in the Oxford classification model. AIC and $\chi^{2}$ of the simplified classification (Beijing classification) were 582 and 114.32, respectively.

A reclassification of the Cox survival regression models is presented (Table 12). The prognostic value of the Lee grade was higher than that of a model containing only established baseline clinical indicators for progression to ESRD or a $\geq 50 \%$ decline in eGFR $(0.50,95 \% \mathrm{CI}$ 0.21-0.74). The prognostic value of the Haas classification compared to that of a model containing only the established baseline clinical parameters was not statistically significant (with a negative value in the $95 \% \mathrm{CI}$ ). The prognostic value of the Oxford classification was higher than that of a model containing only established baseline clinical indicators for progression to ESRD or a $\geq 50 \%$ decline in eGFR $(0.48,95 \%$ CI $0.28-0.71)$. The prognostic value of the Lee grade compared to that of the Oxford classification was not statistically significant (with a negative value in the $95 \% \mathrm{CI}$ ). The prognostic value of the Haas classification was lower than that of the Oxford classification for progression to ESRD or a $\geq 50 \%$ decline in eGFR $(0.59,95 \% \mathrm{CI} 0.10-0.74)$ and that of the Lee grade for progression to ESRD or a $\geq 50 \%$ decline in eGFR $(0.53,95 \%$ CI $0.23-0.92)$. The prognostic value of the simplified classification (Beijing classification) was similar to that of the Oxford classification.

\section{Discussion}

This study confirmed that pathological lesions determined by the Lee grade, the Haas classification, and the Oxford classification showed independent and limited prognostic value in progression to ESRD or a $\geq 50 \%$ decline in eGFR in IgAN. By comparing the NRI of different Cox regression models, this research indicated that both the Lee grade and the Oxford classification showed incremental prognostic value based on established baseline clinical indicators, and that the Haas classification may be slightly inferior to the Lee grade and the Oxford classification. This study provided an objective basis for prognostic value comparison of the Lee grade, the Haas classification, and the Oxford classification in primary IgAN.

Comparison of the prognostic value of different Cox survival regression models, considering completely different risk factors, remains lacking of reliable statistical methods. NRI was introduced in 2008 with the example of the added value of high-density lipoprotein cholesterol level in coronary risk prediction in the Framingham Heart Study [17]. The NRI captures the change in a person's predicted risk and thus translates into a clinically meaningful change in treatment recommendations. In addition, NRI was used in this study and provided a basis for similar research methodology in the future.

The Lee grade and the Haas classification were both single grade. The biggest difference between them was that the Haas classification considered renal interstitial fibrosis or renal tubular atrophy of $>40 \%$ of the renal cortex important independent prognosis factors. Another difference was that focal and segmental glomerular sclerosis was graded separately in the Haas classification. Previous studies have suggested that pathological lesions determined by the Lee grade and the Haas classification should be prognostic factors for IgAN [11, 12]. This study confirmed pathological lesions determined by the Lee grade and the Haas classification as independent prognostic factors for IgAN, and the Lee grade showed increasing statistically significant prognostic value as the grades progressed, although the risk of development of both endpoints for Lee grade III, relative to grades I and II, was not significant. It was partly because Lee grade III, the most common in IgAN, has significant heterogeneity. However, the prognostic values of different subsets of the Haas classification did not differ 
Kidney
Blood Pressure

Research

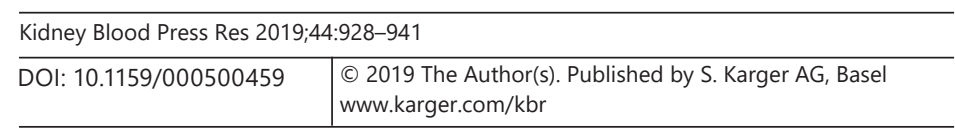

Duan et al.: Comparing Pathological Classifications of IgA Nephropathy

significantly in this study. This study also found that the Lee grade and the Oxford classification, rather than the Haas classification, showed incremental prognostic value based on established baseline clinical indicators. In addition, the Haas classification was slightly inferior to the Lee grade and the Oxford classification. The reason may be that no patients with Haas classifications of I or II reached the primary or secondary endpoints in the study. Merging Haas classifications I, II, and III reduced the prognostic value of the Haas classification.

The Oxford classification, reported in the semiquantitative form, defined pathological lesions for the first time and was simpler than previous semiquantitative grading systems such as the Katafuchi scores. However, the results of the validation of the Oxford classification were inconsistent. This study confirmed that $\mathrm{M}$ and $\mathrm{T}$ lesions of the Oxford classification were independent prognostic factors, while $\mathrm{E}$ and $\mathrm{C}$ lesions were not, which is consistent with a metaanalysis of the validation of the Oxford classification [13] and the VALIGA study [18]. Different from these two studies, E lesion was not an independent prognostic factor, which is consistent with the Oxford cohort [7]. As shown in Table 5, clinicians usually use more active corticosteroids or immunosuppressive agents when patients have $\mathrm{E}$ or $\mathrm{C}$ lesions, which may reverse the degree of pathology and thus weaken the prognostic value of E/C lesions. Other studies [19, 20] also support that the prognostic role of E/C lesions is mainly for patients who do not receive corticosteroids or immunosuppressive agents. Therefore, clinicians should pay more attention to $\mathrm{E}$ and $\mathrm{C}$ lesions when considering the indications of immunosuppressive therapy, which may avoid $\mathrm{E}$ and $\mathrm{C}$ lesions becoming the prognostic factors. Based on active treatment, researchers should devote more attention to $\mathrm{M}$ and $\mathrm{T}$ lesions of the Oxford classification to evaluate prognosis. Other studies also support that $\mathrm{M}$ or $\mathrm{T}$ lesions were key prognostic factors of IgAN [21]. On the other hand, it was hard for clinicians to intuitively assess the prognosis of a patient by the Oxford classification including 4-5 lesions. To solve this problem, we developed a one-dimensional system based on the multivariate Cox regression of the Oxford classification. The new system included four grades, using only $\mathrm{M}$ and $\mathrm{T}$ lesions. Despite using less pathological parameters, the new system (Beijing classification) could provide a prognostic value similar to that of the Oxford classification, which is better than the Haas classification.

The study was designed keeping in mind the prognostic value of different pathological classification systems. Multivariate Cox survival regression models in the study were only adjusted for estimated prognostic clinical indicators at biopsy, such as proteinuria, blood pressure, and kidney function, instead of patients' data during follow-up, which is superior to baseline clinical data in predicting outcomes in IgAN [22]. The results showed that baseline proteinuria, MAP, and levels of eGFR were risk factors for progression to ESRD or a $\geq 50 \%$ decline in eGFR, which is consistent with previous research findings.

This retrospective study involved its own limitations, such as patients not following unified treatment plans. Considering the study sample, emerging prognostic clinical indicators such as smoking status, serum uric acid, cholesterol, and triglyceride were not analyzed in the study. In addition, since IgAN is a chronically progressive disease, the number of patients who progressed to ESRD or showed a $\geq 50 \%$ decline in eGFR in this study, as well as previous similar studies, was not enough to establish significance; therefore, the research outcomes require further validation in future research.

Earlier studies examining the value of prognostic scores in identifying patients at high risk of progressive kidney disease suggest that, even when considering basic histological criteria and clinical data collected over 2 years, clinicians can only explain approximately onethird of the variability in outcome for IgAN patients [19]. The significant heterogeneity and chronic progression of IgAN make it difficult to foretell the outcome for all individual patients at the time of biopsy. Further study of pathological molecular processes may provide an opportunity to improve our understanding of the pathological lesions of this disease and to identify new potential therapeutic targets. 


\section{Kidney \\ Blood Pressure Research}

\begin{tabular}{l|l}
\hline Kidney Blood Press Res 2019;44:928-941 \\
\hline DOI: 10.1159/000500459 & $\begin{array}{l}\text { @ 2019 The Author(s). Published by S. Karger AG, Basel } \\
\text { www.karger.com/kbr }\end{array}$ \\
\hline
\end{tabular}

Duan et al.: Comparing Pathological Classifications of IgA Nephropathy

\section{Conclusion}

The three pathological classification systems of primary IgAN had independent and limited prognostic value in progression to ESRD or a $\geq 50 \%$ decline in eGFR. Both the Lee grade and the Oxford classification showed incremental prognostic value beyond established baseline clinical indicators such as proteinuria, MAP, and eGFR. The Haas classification may be slightly inferior to the Lee grade and the Oxford classification. The hierarchical classification (Beijing classification) using less pathological parameters does not lose predictive efficiency.

\section{Acknowledgments}

Grants (D131100004713003, D181100000118002, D181100000118004) from the Science and Technology Project of Beijing, China; grants (2013BAI09B05, 2015BAI12B06) from the National High Technology Research and Development Program of China.

\section{Statement of Ethics}

Written informed consent was obtained from each patient at the time of renal biopsy, and the study was approved by the PLA General Hospital Clinical Research Ethics Committee. The study was conducted in conformity with the Declaration of Helsinki.

\section{Disclosure Statement}

The authors declare no conflicts of interest.

\section{References}

1 D’Amico G. The commonest glomerulonephritis in the world: IgA nephropathy. Q J Med. 1987 Sep;64(245): 709-27.

2 Woo KT, Chan CM, Chin YM, Choong HL, Tan HK, Foo M, et al. Global evolutionary trend of the prevalence of primary glomerulonephritis over the past three decades. Nephron Clin Pract. 2010;116(4):c337-46.

3 Schena FP. A retrospective analysis of the natural history of primary IgA nephropathy worldwide. Am J Med. 1990 Aug;89(2):209-15.

4 D'Amico G. Natural history of idiopathic IgA nephropathy: role of clinical and histological prognostic factors. Am J Kidney Dis. 2000 Aug;36(2):227-37.

5 Lee SM, Rao VM, Franklin WA, Schiffer MS, Aronson AJ, Spargo BH, et al. IgA nephropathy: morphologic predictors of progressive renal disease. Hum Pathol. 1982 Apr;13(4):314-22.

6 Haas M. Histologic subclassification of IgA nephropathy: a clinicopathologic study of 244 cases. Am J Kidney Dis. 1997 Jun;29(6):829-42.

7 Working Group of the International IgA Nephropathy Network and the Renal Pathology Society, Coppo R, Troyanov S, Camilla R, Hogg RJ, Cattran DC, Cook HT, et al. The Oxford classification of IgA nephropathy: rationale, clinicopathological correlations, and classification. Kidney Int. 2009 Sep;76(5):534-45.

8 Trimarchi H, Barratt J, Cattran DC, Cook HT, Coppo R, Haas M, et al.; IgAN Classification Working Group of the International IgA Nephropathy Network and the Renal Pathology Society; Conference Participants. Oxford Classification of IgA nephropathy 2016: an update from the IgA Nephropathy Classification Working Group. Kidney Int. 2017 May;91(5):1014-21.

9 Kashgarian M, Hayslett JP: Renal involvement in systemic lupus erythematosus. 1994;

10 Working Group of the International IgA Nephropathy Network and the Renal Pathology Society, Coppo R, Troyanov S, Camilla R, Hogg RJ, Cattran DC, Cook HT, et al. The Oxford classification of IgA nephropathy: pathology definitions, correlations, and reproducibility. Kidney Int. 2009 Sep;76(5):546-56. 
11 Frimat L, Briançon S, Hestin D, Aymard B, Renoult E, Huu TC, et al. IgA nephropathy: prognostic classification of end-stage renal failure. L'Association des Néphrologues de l'Est. Nephrol Dial Transplant. 1997 Dec;12(12): 2569-75.

12 Daniel L, Saingra Y, Giorgi R, Bouvier C, Pellissier JF, Berland Y. Tubular lesions determine prognosis of IgA nephropathy. Am J Kidney Dis. 2000 Jan;35(1):13-20.

13 Lv J, Shi S, Xu D, Zhang H, Troyanov S, Cattran DC, et al. Evaluation of the Oxford Classification of IgA nephropathy: a systematic review and meta-analysis. Am J Kidney Dis. 2013 Nov;62(5):891-9.

14 Park KS, Han SH, Kie JH, Nam KH, Lee MJ, Lim BJ, et al. Comparison of the Haas and the Oxford classifications for prediction of renal outcome in patients with IgA nephropathy. Hum Pathol. 2014 Feb;45(2):236-43.

15 Stevens LA, Claybon MA, Schmid CH, Chen J, Horio M, Imai E, et al. Evaluation of the Chronic Kidney Disease Epidemiology Collaboration equation for estimating the glomerular filtration rate in multiple ethnicities. Kidney Int. 2011 Mar;79(5):555-62.

16 Pencina MJ, D'Agostino RB Sr, Steyerberg EW. Extensions of net reclassification improvement calculations to measure usefulness of new biomarkers. Stat Med. 2011 Jan;30(1):11-21.

17 Pencina MJ, D’Agostino RB Sr, D’Agostino RB Jr, Vasan RS. Evaluating the added predictive ability of a new marker: from area under the ROC curve to reclassification and beyond. Stat Med. 2008 Jan;27(2):157-72; discussion 207-12.

18 Coppo R, Troyanov S, Bellur S, Cattran D, Cook HT, Feehally J, et al.; VALIGA study of the ERA-EDTA Immunonephrology Working Group. Validation of the Oxford classification of IgA nephropathy in cohorts with different presentations and treatments. Kidney Int. 2014 Oct;86(4):828-36.

19 Chakera A, MacEwen C, Bellur SS, Chompuk LO, Lunn D, Roberts IS. Prognostic value of endocapillary hypercellularity in IgA nephropathy patients with no immunosuppression. J Nephrol. 2016 Jun;29(3):367-75.

20 Zhang X, Shi S, Ouyang Y, Yang M, Shi M, Pan X, et al. A validation study of crescents in predicting ESRD in patients with IgA nephropathy. J Transl Med. 2018 May;16(1):115.

21 Roberts IS. Pathology of IgA nephropathy. Nat Rev Nephrol. 2014 Aug;10(8):445-54.

22 Bartosik LP, Lajoie G, Sugar L, Cattran DC. Predicting progression in IgA nephropathy. Am J Kidney Dis. 2001 Oct;38(4):728-35. 